\title{
Locating replenishment stations for electric vehicles: Application to Danish traffic data
}

Wen, Min; Laporte, Gilbert; Madsen, Oli B.G. ; Nørrelund, Anders Vedsted; Olsen, Allan

Publication date:

2012

Document Version

Early version, also known as pre-print

Link back to DTU Orbit

Citation (APA):

Wen, M., Laporte, G., Madsen, O. B. G., Nørrelund, A. V., \& Olsen, A. (2012). Locating replenishment stations for electric vehicles: Application to Danish traffic data.

\section{General rights}

Copyright and moral rights for the publications made accessible in the public portal are retained by the authors and/or other copyright owners and it is a condition of accessing publications that users recognise and abide by the legal requirements associated with these rights.

- Users may download and print one copy of any publication from the public portal for the purpose of private study or research.

- You may not further distribute the material or use it for any profit-making activity or commercial gain

- You may freely distribute the URL identifying the publication in the public portal

If you believe that this document breaches copyright please contact us providing details, and we will remove access to the work immediately and investigate your claim 


\title{
Locating Replenishment Stations for Electric Vehicles: Application to Danish Traffic Data
}

\author{
Min Wen ${ }^{1}$, Gilbert Laporte ${ }^{2}$, Oli B.G. Madsen ${ }^{1}$, Anders V. Nørrelund ${ }^{1}$ and Allan Olsen ${ }^{1}$ \\ January 17, 2012
}

\begin{abstract}
${ }^{1}$ Department of Transport, Technical University of Denmark, Bygningstorvet 116 Vest, DK-2800 Kongens Lyngby, Denmark, \{mw@transport.dtu.dk, ogm@transport.dtu.dk, ann@transport.dtu.dk, aol@transport.dtu.dk \}

${ }^{2}$ Canada Research Chair in Distribution Management and CIRRELT, HEC Montréal, 3000 chemin de la Côte-Sainte-Catherine, Montréal, Canada H3T 2A7, gilbert.laporte@cirrelt.ca
\end{abstract}

\begin{abstract}
Environment-friendly electric vehicles have gained substantial attention in governments, industry and universities. The deployment of a network of recharging stations is essential given their limited travel range. This paper considers the problem of locating electronic replenishment stations for electric vehicles on a traffic network with flow-based demand. The objective is to optimize the network performance, for example to maximize the flow covered by a prefixed number of stations, or to minimize the number of stations needed to cover traffic flows. Two mixed integer linear programming formulations are proposed to model the problem. These models are tested on real-life traffic data collected in Denmark. Computational results are presented.
\end{abstract}

\section{Introduction}

During the past few decades, environmental concerns have generated a renewed interest in electrical vehicles. The current yearly worldwide sales of fully electric vehicles now stand at around 20,000 units. The market is expected to grow to 750,000 units in 2020 in the European Union (EU) alone (Philippe (2011)). The 
major advantage of electrical vehicles is that they are more environment-friendly than traditional vehicles. They emit much less $\mathrm{CO}_{2}$ and almost no air pollutants. However, electrical vehicles have a limited driving range due to the low density of their batteries. It is therefore important to supply electrical vehicles with recharging stations to lengthen their autonomy. Around 1000 electric charging stations are now in operation in the USA, distributed in 39 states (U.S. Department of Energy (2011)). It is predicted that the number of public charging points in the EU will grow to close to two million by 2017 (Pike Research (2011)). In Europe, the approximate investment in such infrastructure over the next seven years is likely to be about five billion Euros (Pike Research (2011)). This expenditure will largely be motivated by local government initiatives aimed at boosting the expansion of public charging infrastructures for electric vehicles. It is estimated that more than 4.7 million electric vehicle charge points will be installed worldwide by 2015 (Pike Research (2011)).

Locating stations is a central issue in the deployment of charging infrastructure. In this work, we investigate the problem of locating electronic replenishment stations for electric vehicles on the basis of traffic flows. Two models will be developed and compared. In the first one, the aim is to maximize the total flow captured by a given number of stations. In the second model the aim is to minimize the number of stations required to capture the entire flow. In both cases we impose a driving range constraint which ensures that every stretch of at least $r \mathrm{~km}$ is covered by at least one station. The problem belongs to the family of Flow Interception Facility Location Problems (FIFLPs) in which demand is expressed by origin-destination (OD) flows on a directed network. It is assumed that drivers can always replenish at their origin or at their destination. This means that replenishment is not an issue on OD paths not exceeding $r \mathrm{~km}$.

The FIFLP was introduced by Hodgson (1981) in relation with the location of daycare facilities. Over the past 30 years, different models have been proposed to characterize a wide range of applications, such as the location of police inspection stations (Hodgson et al. (1996), Gendreau et al. (2000) and Selmić et al. (2010)), of road detecting sensors (Liu and Danczyk (2009)), of gas and refueling stations (Kuby et al. (2009), Lim and Kuby (2010), Upchurch et al. (2009), Wang and Lin (2009) and Wang and Wang (2010)), and so on. Boccia et al. (2009) provide a review on the problems, models and methods for FIFLPs.

The main contribution of this paper is the development of two models for the Electronic Replenishment Station Location Problem (ERSLP) and their assessment on real-life traffic data collected in Denmark. The remainder of the paper is organized as follows. A formal problem description and two mathematical models 
are provided in Section 2. The data and a data preprocessing algorithm and the computational results are presented in Section 3, followed by conclusions in Section 4.

\section{Formal problem description and mathematical mod- els}

A natual way to model the ERSLP is to use a flow capturing formulation. However, as rightly noted by Lim and Kuby (2010), the ERSLP is not a standard FIFPL because several facilities may be required to cover a flow. Several formulations are possible, depending on how the variables and constraints are defined. Lim and Kuby (2010) have developed a model which requires the enumeration of all facility combinations. Here we develop a more parsimonious but equivalent model applicable to relatively large scale applications. Our model works with a directed graph $G=(V, A)$, where $V$ is a set of nodes and $A=\{(i, j): i, j \in V, i \neq j\}$ is a set of arcs. A node can represent the origin or the destination of an OD path or potential location sites for stations. The set of OD paths is denoted by $P$. Let $f_{p}$ be the traffic flow of path $p$. Each path $p \in P$ is represented by an ordered sequence of nodes $V^{p}=\left(v_{1}^{p}, \ldots, v_{l}^{p}\right)$. Different paths can share some nodes. The set of all nodes is $V=V^{1} \cup \ldots \cup V^{|P|}$. We denote by $t_{i j}$ the length of a shortest path from $i$ to $j$. The driving range of a vehicle is the maximal distance it can drive without replenishing and is denoted by $r$. We denote by $S_{i p}$ the maximal ordered subset $\{i, \ldots, h\}$ of a subpath $p$, starting at node $i$, that can be driven without replenishment, i.e., such that $t_{i h} \leq r$.

\subsection{Maximal flow capture model}

In the maximal flow capture model (MFCM), the aim is to maximize the captured flow using $m$ stations, subject to the driving range constraint. This model uses binary variables $y_{j}$ equal to 1 if and only if a station is located at node $j$, binary variables $z_{p}$ equal to 1 if and only if the flow of OD path $p$ is covered by sufficient number of stations. To reduce the size of the models, we only consider those OD paths whose length exceeds $r$ and undominated paths: path $p$ dominates path $p^{\prime}$ if $V^{p^{\prime}} \subseteq V^{p}$. Let $P^{\prime}$ be the resulting set of paths. The MFCM is as follows: 
MFCM:

$$
\begin{aligned}
& \operatorname{maximize} \sum_{p \in P^{\prime}} f_{p} z_{p} \\
& \text { such that } \sum_{j \in V} y_{j}=m \\
& \sum_{j \in S_{i p}} y_{j} \geq z_{p} \quad \forall p \in P^{\prime}, i \in V^{p} \\
& y_{j} \in\{0,1\} \quad \forall j \in V \\
& z_{p} \in\{0,1\} \quad \forall p \in P^{\prime} \text {. }
\end{aligned}
$$

In this formulation, the objective maximizes the traffic covered by the stations. Constraints (2) impose the location of $m$ stations. They ensure that no feasible solution is excluded since all locations in $V$ are considered as candidates. Constraints (3) state that every subpath of an OD path $p$ that is covered (i.e. $z_{p}=1$ ) contains a replenishment station within $r$ units of each of its nodes. Constraints (4) and (5) define the binary variables.

\subsection{Total flow capture model}

In the total flow capture model (TFCM), the aim is to cover all traffic with the least number of stations. The model is

TFCM:

$$
\begin{array}{ll}
\operatorname{minimize} & \sum_{j \in V} y_{j} \\
\text { such that } & \sum_{j \in S_{i p}} y_{j} \geq 1 \quad \forall p \in P^{\prime}, i \in V^{p} \\
& y_{j} \in\{0,1\} \quad \forall j \in V .
\end{array}
$$

In this model, constraints (7) ensure that each OD path is covered by at least one station and that the driving range constraints are satisfied.

\section{Computational results}

Solving MFCM or TFCM requires traffic data. In Denmark which serves as a basis for this study, such data are provided by the government which conducts traffic surveys every 10 years. These surveys include Daily Trip Schedules which describe 


\begin{tabular}{|c|c|}
\hline Number of paths & Length interval \\
\hline \hline 186 & $0 \mathrm{~km}-49 \mathrm{~km}$ \\
\hline 354 & $50 \mathrm{~km}-99 \mathrm{~km}$ \\
\hline 667 & $100 \mathrm{~km}-159 \mathrm{~km}$ \\
\hline 330 & $160 \mathrm{~km}-199 \mathrm{~km}$ \\
\hline 421 & $200 \mathrm{~km}-299 \mathrm{~km}$ \\
\hline 254 & $300 \mathrm{~km}-499 \mathrm{~km}$ \\
\hline 57 & $500 \mathrm{~km}-1210 \mathrm{~km}$ \\
\hline
\end{tabular}

Table 1: Path length distribution

\begin{tabular}{|c|c|}
\hline Number of nodes & Degree interval \\
\hline \hline 50 & $300-530$ \\
\hline 171 & $200-299$ \\
\hline 229 & $150-199$ \\
\hline 647 & $100-149$ \\
\hline 278 & $80-90$ \\
\hline 1443 & $50-80$ \\
\hline 7407 & $20-49$ \\
\hline 11,387 & $10-19$ \\
\hline 84,246 & $1-9$ \\
\hline
\end{tabular}

Table 2: Node degree distribution

the OD paths of each interviewed person for a given day. These paths are then transformed into shortest path by means of an algorithm based on a route choice model. Every shortest path is represented by a sequence of nodes including junctions, highway entry and exit points, and other potential locations for stations. Figure 1 depicts a shortest path from Copenhagen to Aarhus.

In the traffic data, there are 2269 paths of varying lengths and 105,827 nodes. Table 1 gives the distribution of path lengths of paths and Table 2 gives the distribution of node degrees. The degree of node represents the number of paths covering it. The average node degree is 8.7.

To reduce the instance size, we preprocess the data by removing nodes that are only covered by a relatively small number of paths and that are located close to other nodes. Two parameters are used to control the degree of reduction in the instance size. One is called "LBDegree": only those nodes with a degree less than LBDegree will be considered to be removed from the paths. The other parameter is called "LBDist". We only remove a node from a path if the distances between its predecessor and its successor is shorter than LBDist. This is to prevent removing all nodes from a path. The preprocessing algorithm is given in Algorithm 1. It is implemented in $\mathrm{C} \#$. Table 3 gives the number of nodes in the reduced instance 

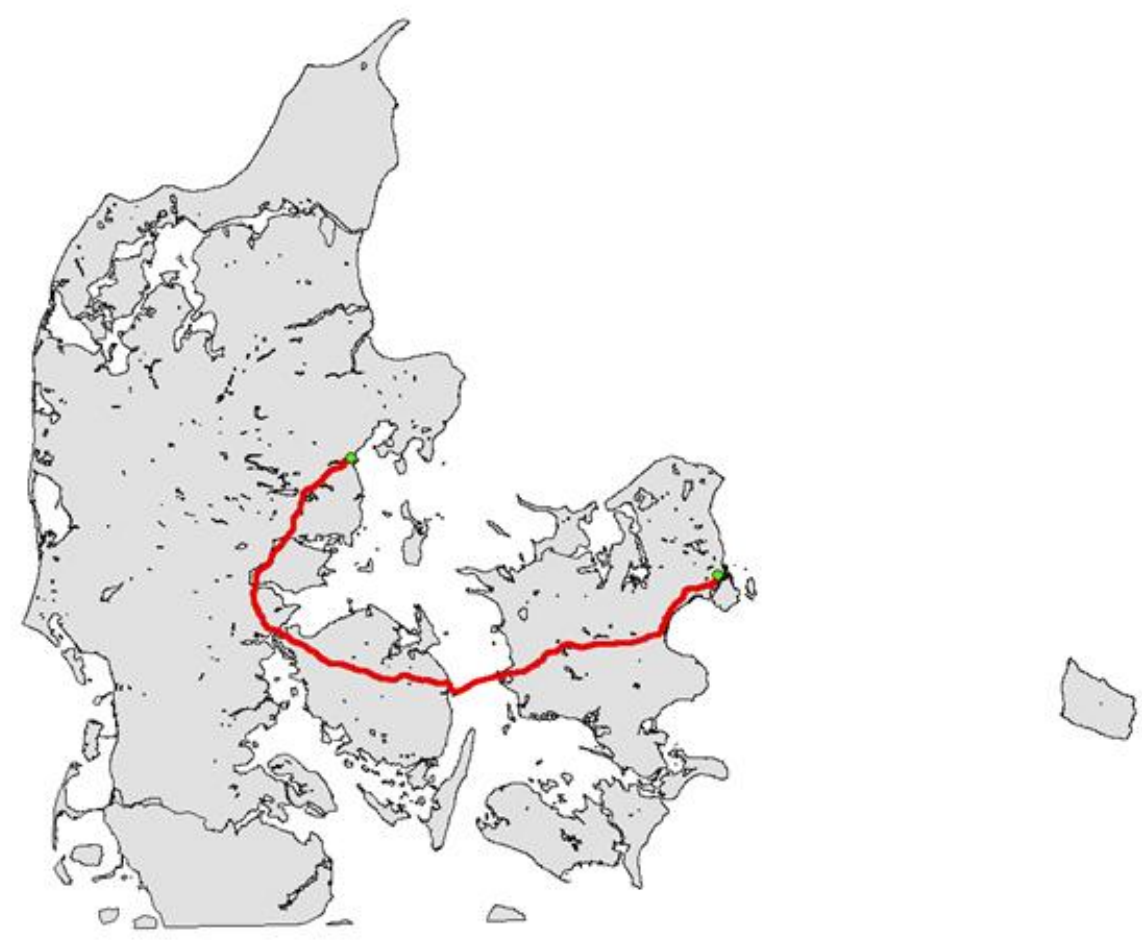

(a) Shortest path from Copenhagen to Aarhus.
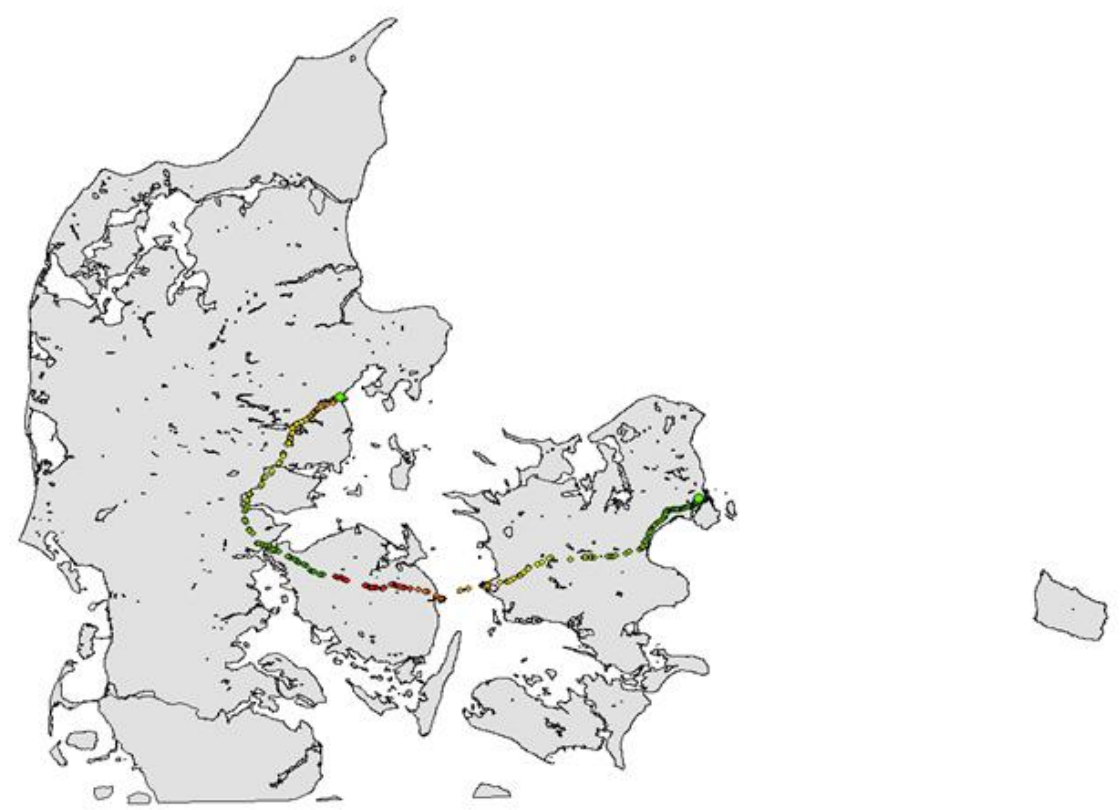

(b) Nodes in the shortest path from Copenhagen to Aarhus.

Figure 1: Shortest path and potential station locations for a given OD pair between Copenhagen and Aarhus 


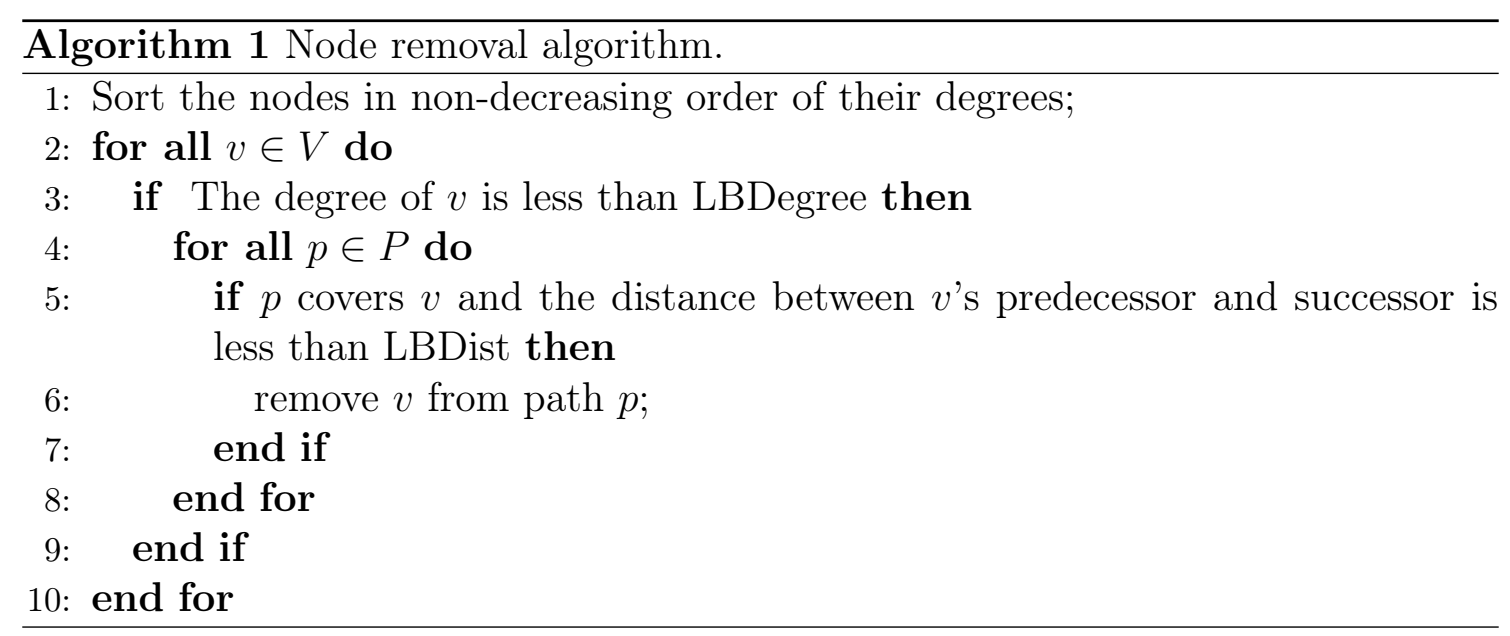

\begin{tabular}{|c|c|c|c|c|}
\hline LBDegree $\backslash$ LBDist $(\mathrm{m})$ & 5000 & 10000 & 20000 & 30000 \\
\hline \hline 0 & 105,827 & 105,827 & 105,827 & 105,827 \\
\hline 3 & 58,001 & 57,276 & 57,136 & 57,124 \\
\hline 5 & 41,270 & 39,996 & 39,646 & 39,603 \\
\hline 10 & 24,725 & 22,704 & 21,893 & 21,705 \\
\hline 50 & 8937 & 5865 & 4315 & 3734 \\
\hline 80 & 7941 & 4786 & 3192 & 2547 \\
\hline 100 & 7808 & 4644 & 3022 & 2368 \\
\hline 150 & 7394 & 4209 & 2567 & 1887 \\
\hline 1000 & 7219 & 3990 & 2313 & 1615 \\
\hline
\end{tabular}

Table 3: Number of nodes in the reduced instances for different parameter settings of (LBDegree, LBDist).

for different values of (LBDegree, LBDist). With LBDist $=5000 \mathrm{~m}$, by increasing LBDegree from 0 to 50 , the instance size is reduced by more than $90 \%$, but it cannot be further reduced significantly when LBDegree is greater than 50. However, increasing LBDist can still reduce instance size when LBDegree $>50$. When LBDegree $=1000$, which means that any node can be removed as long as the LBDist requirement is satisfied, the instance size can still be reduced by $75 \%$ by increasing LBDist from $5 \mathrm{~km}$ to $30 \mathrm{~km}$.

\subsection{Results of the maximal flow capture model}

The MFCM model was tested with different values of $m(5,10,50,80$ and 100) on the reduced instances generated by different parameter settings (LBDegree, LBDist). The results are provided in Table 4 . For each reduced instance, the first row shows the number of paths covered when $m$ is 5, 10, 50, 80 and 100, respectively, and the second row shows the corresponding percentage. The last row gives the running time. For example, for the reduced instance generated by the parameter setting 
(150, 20,000), there are 469, 661, 1009, 1060 and 1062 paths covered by the selected 5, 10, 50, 80 and 100 stations, respectively. These paths correspond to 44, 62, 95, 99 and 100 percent of all the paths. The corresponding running times are 70, 349, 201, 82,864 and 6 seconds. Figure 2 illustrates the locations of stations on the map of Denmark for the reduced instance generated by the parameter setting $(150,20,000)$. The lines on the map are roads with major traffic flows in Denmark.

In general, the more the instance size is reduced, the shorter the running time is and the worse the solution quality is. Comparing the reduced instance generated by the parameter setting $(150,10,000)$ with the problem by the parameter setting $(1000,30,000)$, we can see that the instance size is reduced by $62 \%$, the running time is shortened by more than $83 \%$ and the solution deteriorates by less than $9 \%$. From the table, the parameter setting $(150,30,000)$ seems to provide a good trade-off between computational time and solution quality.

\subsection{Results of the total flow capture model}

In the TFCM, we have set the driving range to $160 \mathrm{~km}$. The model was run on different reduced instances. The results are shown in Table 5. For each test, the minimum number of stations needed, the size of the model and the running time are given. For example, for the reduced instance generated by the parameter setting $(150,10,000)$, at least 68 stations are needed. There are 4209 variables and 46,906 constraints in the model, and it takes 4987 seconds to solve it. The locations of the 68 stations are depicted in Figure 3. In general, the more the instance size is reduced, the larger is the number of stations needed.

\section{Conclusions}

The deployment of charging infrastructure is important for electric vehicles due to their short travel range. We have considered the problem of locating electrical replenishment stations in Denmark based on data collected over the past ten years. To meet different criteria, we have presented two models. In the first model, the coverage of paths is maximized given a fixed number of stations. In the second model, the travel range is considered explicitly and the objective is to minimize the number of stations needed to supply enough endurance to the vehicles on long trips.

We have preprocessed the data to reduce the instance size and thus decrease computation time. Two parameters were used to control the aggregation level. Solutions to the reduced instances of different sizes were presented. It was found 


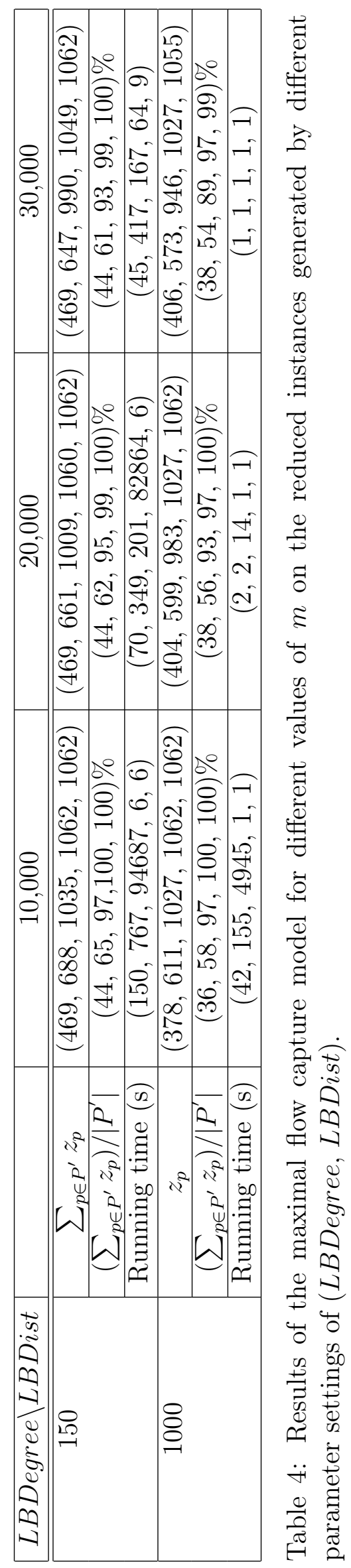



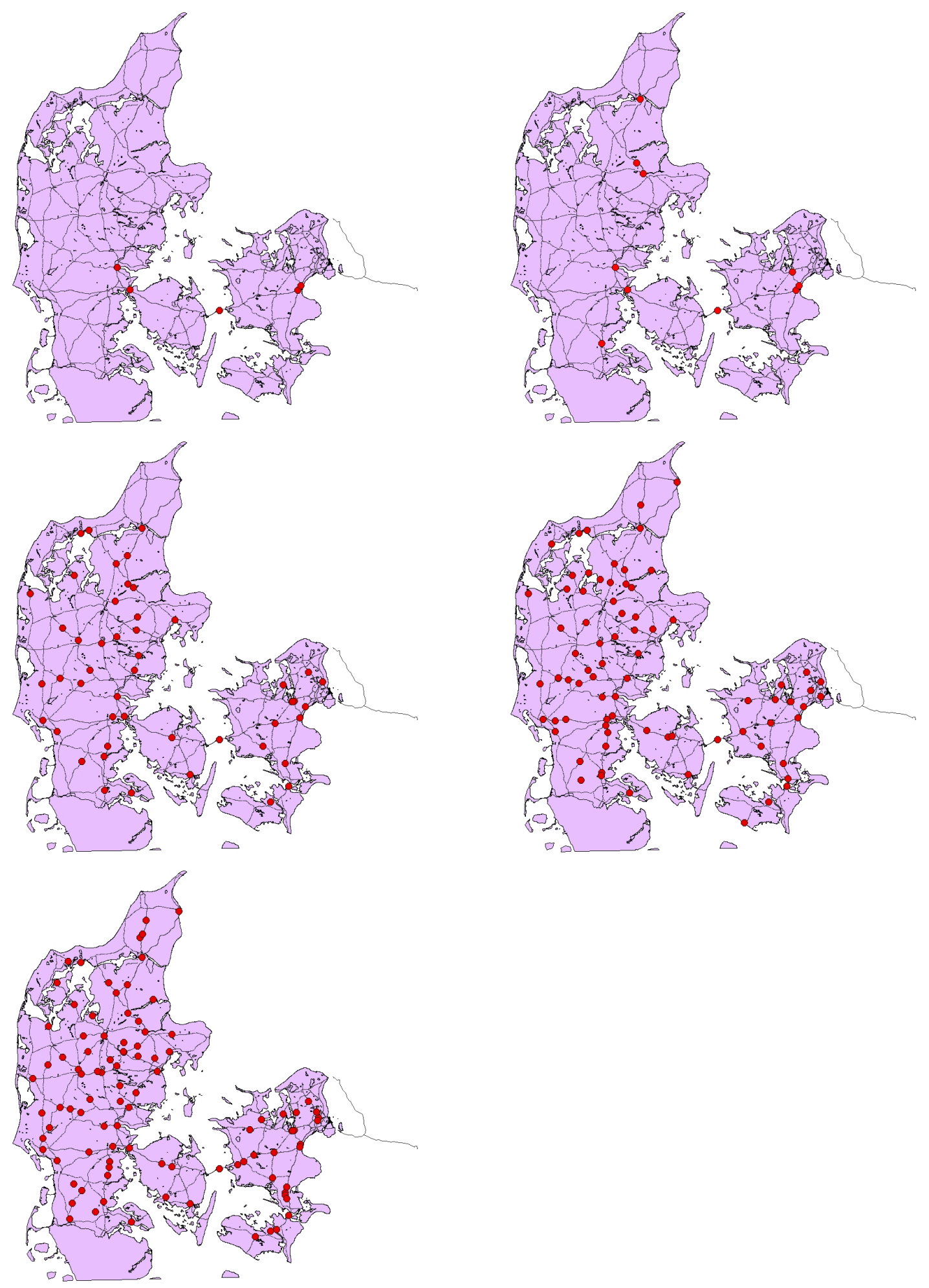

Figure 2: The locations of the stations for $m=5,10,50,80$ and 100 in the solution to the reduced instance generated by the parameter setting (LBDegree $=150$, LBDist $=20,000)$. 


\begin{tabular}{|c|c|c|c|}
\hline LBDegree $\backslash$ LBDist & 10,000 & 20,000 & 30,000 \\
\hline \multirow{4}{*}{150} & 68 & 82 & 93 \\
\cline { 2 - 4 } & 4209 variables & 2566 variables & 1887 variables \\
& 46,906 constraints & 42,449 constraints & 41,055 constraints \\
\cline { 2 - 4 } & time $=4987 \mathrm{~s}$ & time $=640 \mathrm{~s}$ & time $=7 \mathrm{~s}$ \\
\hline \multirow{3}{*}{1000} & 70 & 90 & 107 \\
\cline { 2 - 4 } & 3990 variables & 2313 variables & 1615 variables \\
& 16,264 constraints & 8274 constraints & 5521 constraints \\
\cline { 2 - 4 } & time $=1700 \mathrm{~s}$ & time $=2.17 \mathrm{~s}$ & time $=0.3 \mathrm{~s}$ \\
\hline
\end{tabular}

Table 5: Results of the total flow capture model on different reduced instances generated by different parameter settings of (LBDegree, LBDist).

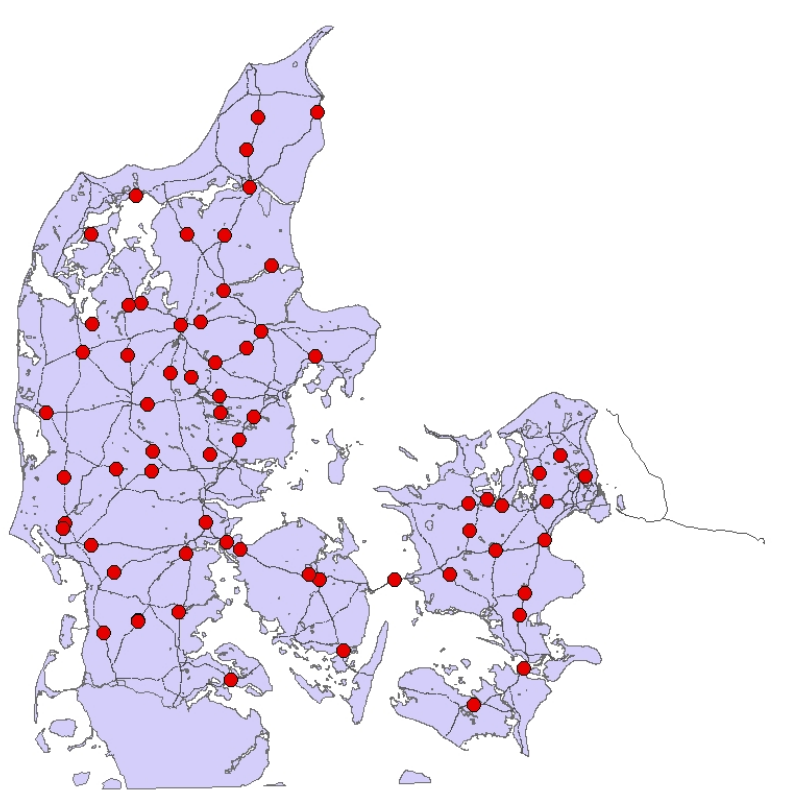

Figure 3: Locations of the 68 stations in the solution to the reduced instance generated with the parameter setting $($ LBDegree $=150$, LBDist $=10,000)$. 
that the more the instance size is reduced, the larger is the number of stations needed and 68 stations are sufficient to ensure the driving range for the long paths.

In the future, more accurate models consisting of more real-life constraints and objectives can be investigated. Fast metaheuristic, e.g., adaptive large neighbourhood search, can also be developed to solve large scale problems.

\section{Acknowledgements}

This work was partly supported by the Canadian Natural Sciences and Engineering Research Council under grants 227837-04 and 39682-10. This support is gratefully acknowledged.

\section{References}

Boccia, M., Sforza, A., and Sterle, C. (2009). Flow intercepting facility location: Problems, models and heuristics. Journal of Mathematical Modelling and Algorithms, 8(1):35-79.

Gendreau, M., Laporte, G., and Parent, I. (2000). Heuristics for the location of inspection stations on a network. Naval Research Logistics, 47(4):287-303.

Hodgson, M, J. (1981). The location of public facilities intermediate to the journey to work. European Journal of Operational Research, 6(2):199-204.

Hodgson, M, J., Rosing, K, E., and Zhang, J, J. (1996). Locating vehicle inspection stations to protect a transportation network. Geographical Analysis, 28(4):299314.

Kuby, M., Lines, L., Schultz, R., Xie, Z., Kim, J.-G., and Lim, S. (2009). Optimization of hydrogen stations in Florida using the Flow-Refueling Location Model. International Journal of Hydrogen Energy, 34(15):6045-6064.

Lim, S. and Kuby, M. (2010). Heuristic algorithms for siting alternative-fuel stations using the Flow-Refueling Location Model. European Journal of Operational Research, 204(1):51-61.

Liu, H, X. and Danczyk, A. (2009). Optimal sensor location for freeway bottleneck identification. Computer-Aided Civil and Infrastructure Engineering, 24(8):535550 . 
Philippe, J. (2011). European policy on electric vehicles. Conference on Electric vehicles - Challenges of the New Mobility, Sofia.

Pike Research (2011). Strategic technology and market analysis of electric vehicle charging infrastructure in Europe. http://www.reportlinker.com/p0503640/ Strategic-Technology-and-Market-Analysis-of-Electric-Vehicle-ChargingInfrastructure-in-Europe.pdf.

Selmić, M., Teodorović, D., and Vukadinović, K. (2010). Locating inspection facilities in traffic networks: an artificial intelligence approach. Transportation Planning and Technology, 33(6):481-493.

Upchurch, C., Kuby, M., and Lim, S. (2009). A model for location of capacitated alternative-fuel stations. Geographical Analysis, 41(1):85-106.

U.S. Department of Energy (2011). Alternative fueling station total counts by state and fuel type. http://www.afdc.energy.gov/afdc/fuels/stations_counts.html.

Wang, Y.-W. and Lin, C.-C. (2009). Locating road-vehicle refueling stations. Transportation Research Part E - Logistics and Transportation Review, 45(5):821-829.

Wang, Y.-W. and Wang, C.-R. (2010). Locating passenger vehicle refueling stations. Transportation Research Part E - Logistics and Transportation Review, 46(5):791801. 